\title{
Clinical aspects of satellite diseases and cricoarytenoid joint involvement. Arthritis of the cricoarytenoid joint. Clinical case.
}

\author{
Topalova-Shishmanova A. ', Dzhambazov K. ${ }^{1}$, Konsulov Sp. ${ }^{1}$, Traikova N. ${ }^{2}$, \\ Anesteva R. ${ }^{1}$, Kraeva M. ${ }^{1}$ \\ Department of Otorhinolaryngology' \\ Department of Radiology ${ }^{2}$ \\ UMHAT "St. George", Plovdiv \\ Medical University of Plovdiv, Bulgaria
}

\begin{abstract}
Introduction: Laryngeal involvement in rheumatoid arthritis is a rarely mentioned complication in clinical practice. In the literature over the last 10 years, single articles on the subject have been published, presenting a small number of clinical cases. Cricoarytenoid arthritis is a life-threatening condition requiring timely anesthesia and otorhinolaryngological intervention, where the tracheostomy has an important therapeutic place.

Aim: With the presentation of our clinical case we aim to expand the knowledge about systemic diseases and their possible acute manifestation in otorhinolaryngological practice.

Materials and methods: We present a clinical case of patient M.S., 63 years old, female, with a long medical history of rheumatoid arthritis. She was admitted to the clinic with a tracheostomy cannula placed for acute laryngeal dyspnea. Physical examination with a fibrolaryngoscope revealed bilateral paresis of the vocal folds, in a medial position, without phonatory and respiratory activity. Computer tomography of the neck, chest, with a focus on the mediastinum - without clinical data on tumor processes. Laboratory tests reveal a repeatedly elevated rheumatoid factor.

Results: MRI revealed a collapse of the larynx, with pronounced fatty degeneration of the thyroarytenoid and cricoarytenoid muscles. The patient was referred to a rheumatologist to control the chronic exacerbation process, with a definitive tracheostomy cannula.

Conclusion: Pathological changes in the cricoarytenoid joint in patients with rheumatoid arthritis, as well as scleroderma, lupus, Felty's syndrome, Tiez syndrome and other systemic diseases, are a common finding, although diagnosed in the past at autopsy. It can be acute, subacute and chronic, but in otorhinolaryngological practice it occupies an important clinical place in emergencies.

Key words: cricoarytenoid joint, rheumatoid arthritis, tracheostomy
\end{abstract}

\section{Introduction}

Rheumatoid arthritis (RA) is a chronic inflammatory joint disease that involves the cartilage and bone of small and medium-sized joints, the main clinical manifestation of which is erosive-destructive progressive polyarthritis. May affect extra-articular tissues and organs.

Rheumatoid arthritis is one of the most common rheumatic diseases. It affects about $1 \%$ of the world's population with certain regional differences. In southern European countries, an average of 3 per 10,000 people are affected, and in northern European countries the incidence is 5 per 10,000 . The female / male ratio is $6 / 1$ to 60 years of age, with the incidence equalizing that age.

The etiopathogenesis of rheumatoid arthritis is unclear. Its occurrence is determined by the interaction of genetic, immunological and environmental factors. It is an immune dysregulation that leads to chronic inflammation and causes organ disorders, increased morbidity and mortality. One of the most important signs of immune dysregulation is the activation of inflammatory cytokines, such as IL10, IL6 or TNF-alpha. Their effects lead to pathological changes.

The temporomandibular joint, larynx, cervical spine and audiovestibular system are sites of involvement in the head and neck region of patients with RA. ${ }^{6}$ 
In RA, the larynx is involved in $27-80.9 \%$ of patients, varying in different studies $2,3,4$. Its involvement in the early course of the disease is in most cases underestimated due to the prevalence of systemic manifestations, inconspicuous clinical findings on the part of the larynx and non-specific symptoms.

Laryngoscopic examination may not reveal any findings, but mucosal edema and diffuse or localized inflammation may also be seen. Other laryngoscopic findings of laryngeal involvement include cricoarytenoid joint involvement, interarytenoid fibrosis, vocal cord paralysis, and rheumatoid nodule formation.?

Patients with RA are subject to screening for laryngeal involvement, using subjective and objective methods. A subjective VHI (voice handicap index) questionnaire and objective acoustic sound analysis are used in the diagnostic algorithm.

As the disease progresses, acute airway obstruction may occur, which is a life-threatening condition requiring timely anesthesia and otorhinolaryngological intervention, where the tracheostomy occupies an important clinical place.

\section{Material and methods}

This is a 63-year-old woman diagnosed with seropositive RA in 2004. She underwent sequential treatment with a synthetic pathogen Methotrexate $10 \mathrm{mg}$. weekly, biological disease-modifying antirheumatic agents (Remicade, Humira, RoActemra) and last with Clisia until 2018, when therapy was discontinued due to the appearance of Herpes Zoster.

The patient was admitted to the clinic with a tracheostomy cannula placed on the occasion of acute laryngeal dyspnea in another hospital. There is a history of intermittent voice loss and difficulty eating solid foods.

As concomitant diseases - Rheumatoid arthritis, Gastric ulcer, Iron deficiency anemia, Struma nodosis.

To make the diagnosis, the following tests were performed - complete blood count, general physical (Fig. 1) and otorhinolaryngological examination, fibrolaryngoscopy, computed tomography, magnetic resonance imaging.

\section{Results}

Indirect laryngoscopy and fibrolaryngoscopy - bilateral paresis of the vocal cords, in the medial position, without phonatory and respiratory activity.
From blood tests - CBC - Anemic syndrome; no leukocytosis; thrombocytosis; ESR - $77 \mathrm{~mm} / \mathrm{h}$; $\mathrm{CRP}-55 \mathrm{mg} / \mathrm{L}$, multiple rheumatoid factor - RF - 1881.4 IU / ml (0-20)

$\mathrm{CT}$ of the neck and chest was performed, focusing on the mediastinum - without clinical evidence of a tumor process (Fig. 2).

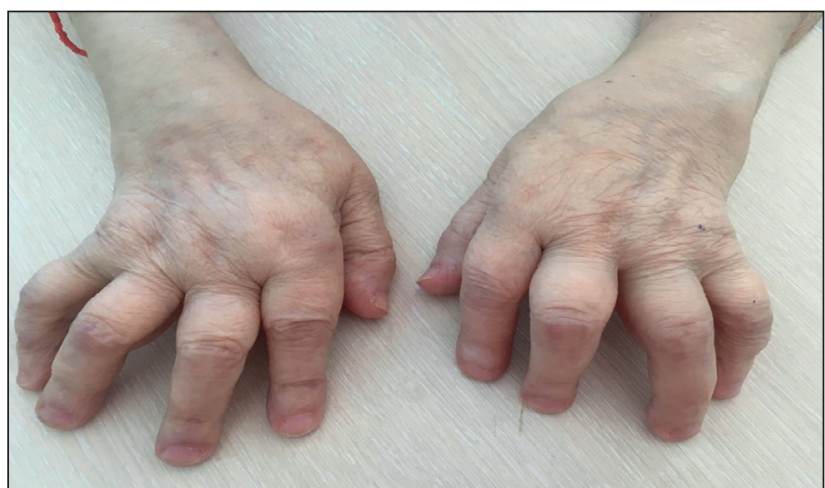

Fig.1. The physical examination reveals deformation and swelling of the metacarpophalangeal joints and limited mobility of the elbow joints, wrists, knees and ankles.

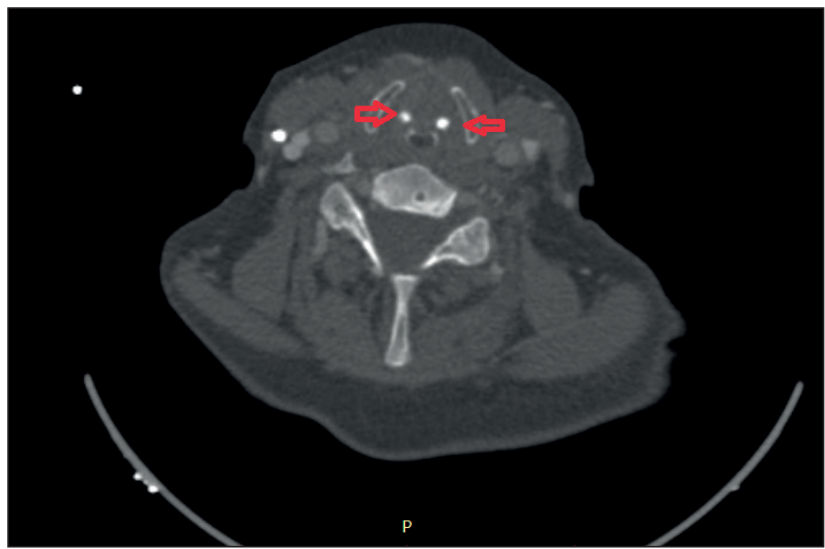

Fig. 2. CT finding for the presence of calcifications in the area of the arytenoids

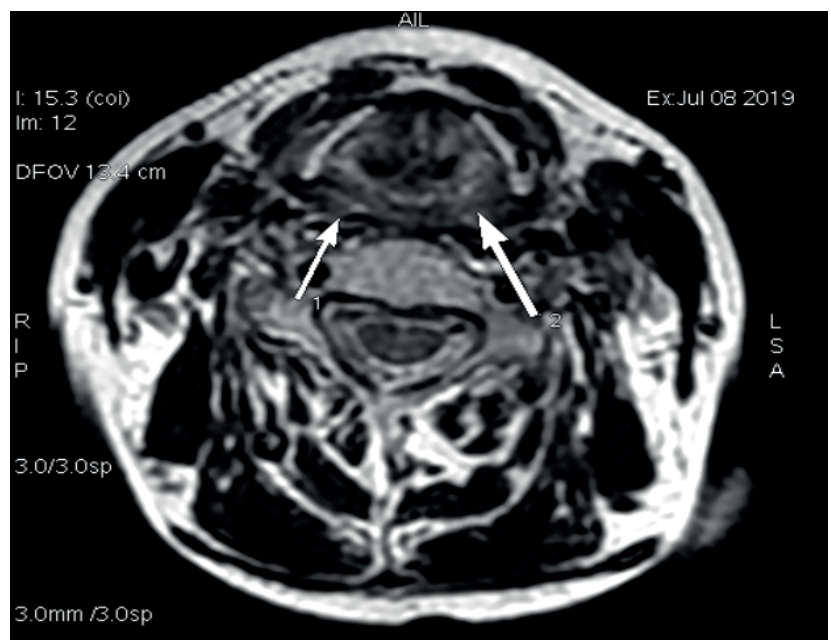

Fig. 3. MRI reveals collapse of the larynx with pronounced fatty degeneration of the thyroarytenoid and cricoarytenoid muscles 
The patient was referred to a rheumatologist to control the chronic exacerbation process, with a definitive tracheostomy cannula.

The possibility of performing an arytenoidectomy was not excluded in the discussion of the patient's therapeutic behavior.

\section{Discussion}

Involvement of the larynx in rheumatoid arthritis was first described by Sir Morell MacKenzie in $1880^{1}$.

Grosmann et al. reported that five of 11 RA patients had laryngeal involvement in post-mortem examination and only two of them were symptomatic. They found that half patients with RA had laryngeal symptoms 5 . Similarly, several studies have reported that up to $50 \%$ of patients are having laryngeal involvement as the sole manifestation of this disease..$^{9,10}$

Pathological changes in the cricoarytenoid joint are most common in patients with rheumatoid arthritis, but can also occur in other conditions such as scleroderma, lupus, gout, Tiez syndrome, Felty syndrome.

Laryngoscopic findings in RA may also include the presence of Bamboo nodes. Bamboo nodes were initially described by Hosako et al. in a female patient with lupus erythematous. Endoscopic visualization shows transversally arranged cystic yellowish bamboo nodes in the submucosal space of the middle portion of the vocal folds. Similar to other laryngeal lesions in patients with RA, these nodes are more often seen in patients with active disease rather than inactive and correlates with antibody deposits ${ }^{11}$, These lesions are seen more commonly in females with history of phonotraumatic behavior and gastroesophageal reflux disease.

In patients with long-standing rheumatoid arthritis, signs of laryngeal involvement may go unnoticed, especially with slow progression.

There are several possible causes for the development of fibrosis and ankylosis of the joint.

The impaired movement may be in the vertical, anteroposterior, or mediolateral directions.

Histopathologic findings vary from inflammatory changes to synovial proliferation and destruction of the articular cartilage, with or without pannus. A second cause for the impaired mobility of the vocal folds is the presence of rheumatoid nodule in either the vocalis muscle and/or near the CAJ hindering its mobility. Erbet al. have shown the presence of these confluent nodules in conjunction with inflammation of the synovial joints, destruction of the laryngeal cartilages, and impinging on the airway ${ }^{12}$. A third cause for the impaired mobility is abductor muscle paralysis. Darke et al. have reported the presence of severe demyelination and degeneration of the recurrent laryngeal and vagus nerves together with atrophy of the

laryngeal muscles with or without obliterative arteritis of the vasa vasorum ${ }^{13}$. A fourth possible etiology is cervicomedullary compression due to rheumatoid involvement of the cervical spine. Link et al. have reported this often overlooked cause of vocal fold palsy in patients with $\mathrm{RA}^{14}$.Watkinson has also described laryngeal amyloidosis as a rare cause of stridor in patients with RA.

Complications of RA may begin to develop within a few months of the onset of clinical symptoms such as acute respiratory failure may be provoked by bacterial infections of the larynx, mechanical lesions of the larynx, or exacerbation of cricoarytenoid arthritis.

Pathological changes in patients with RA create difficulties and pose a risk in endotracheal intubation, which in turn suggests the need for an otolaryngologist and anesthesiologist, with an assessment of performing a tracheostomy.

Table 1. Types of cricoarytenoid arthritis

\begin{tabular}{|l|l|}
\hline $\begin{array}{l}\text { Acute cricoarytenoid } \\
\text { arthritis }\end{array}$ & $\begin{array}{l}\text { Chronic cricoarytenoid } \\
\text { arthritis }\end{array}$ \\
\hline - Feeling of a foreign body & $\begin{array}{l}\text { - Hoarse voice } \\
\text { - Stridor on exertion }\end{array}$ \\
- Tension in the neck & - Shortness of breath \\
- Hoarse voice & - Odinophony \\
- Odynophagia & - Swelling of the neck \\
- Odino / Dysphagia & \\
- Otalgia & \\
- Shortness of breath & \\
- Cough & \\
\hline
\end{tabular}

\section{Treatment}

A multidisciplinary approach involving a rheumatologist, ENT and rehabilitator is needed.

The main principles of treatment are:

- Active treatment in patients with poor prognosis - those with polyarticular involvement, progressive course and high rheumatoid factors.

- The basic agents Methotrexat and Sulphsalazine are preferred.

- Kerstens et al. have reported accelerated nodu- 
Table 2. Clinical signs of cricoarytenoid joint involvement.

\begin{tabular}{|l|l|l|}
\hline Laryngotracheal symptoms & Laryngeal findings & $\begin{array}{l}\text { High-resolution computerized } \\
\text { tomographic findings }\end{array}$ \\
\hline Phonatory symptoms & - Edema & - Cricoarytenoid prominence \\
- Hoarseness & - Hyperemia & Density changes in CAJ* and/or \\
- Breathiness & - Myositis \\
- Vocal fatigue & - Impaired mobility of the vocal fold & - Volume changes in CAJ and/or CTJ \\
- Inability to project the voice & - Fixed vocal cords & Soft tissue changes in CAJ or CTJ \\
- Complete aphonia & - Epiglottitis & Erosion of the CAJ or CTJ \\
Pharyngeal symptoms & - Rheumatic nodules & Ankylosis of the CAJ or CTJ \\
- Dysphagia & - Bamboo nodes & \\
- Odynophagia & & \\
- Sore throat & & \\
- Foreign body sensation & & \\
- Globus pharyngeus & & \\
Airway symptoms & & \\
- Shortness of breath & & \\
- Stridor & & \\
- Dyspnea & & \\
\hline
\end{tabular}

${ }^{*} \mathrm{CAJ}$ : cricoarytenoid joint. *CTJ: cricothyroid joint

Hamdan AL, Sarieddine D. Laryngeal manifestations of rheumatoid arthritis. Autoimmune Dis. 2013; 103081

losis in 5-10\% of patients with RA treated with low-dose methotrexate therapy ${ }^{15}$.With respect to the Bamboo nodes, these lesions may be treated either surgically or conservatively. Hilgert et al. favor conservative approach to these lesions and have reported good outcome with logopedic therapy ${ }^{16}$

- Biological agents are used for those who are not affected by the basic medications.

Patients with RA diagnosed with cricoarytenoid involvement usually do not require additional treatment.

- Some authors reported administration of high doses of steroids systemically or locally into the cricoarytenoid joint. Injection of the cricoarytenoid joint with steroid may be an adjunctive procedure to parental corticosteroid therapy ${ }^{14}$

- In cases of acute respiratory distress of life threatening emergencies, a tracheostomy should be performed to secure the airway.

\section{Conclusion}

RA is a systemic autoimmune disease with widespread clinical manifestations. Otolaryngological signs and symptoms are variable and may not always be properly defined. The appearance of even discrete upper respiratory symptoms in patients with RA should sharpen the clinician's attention to engaging the laryngeal structures.

In these cases, a thorough examination of the ENT organs is recommended in order to exclude cricoarytenoid pathology.

A multidisciplinary approach is needed to ensure adequate laryngeal rehabilitation and alleviate the patient's suffering. 


\section{References}

1. MacKenzie M. Disease of the pharynx, larynx, and trachea. New York: William Wood;1880:347

2. Geterud A, Bake B, Berthelsen B, Bjelle A, Ejnell H. Laryngeal involvement in rheumatoid arthritis. Acta Otolaryngol. 1991; 111: 990-8.

3. Lawry GV, Finerman ML, Hanafee WN, Mancuso AA, Fan PT, Bluestone R. Laryngeal involvement in rheumatoid arthritis. A clinical, laryngoscopic, and computerized tomographic study. Arthritis Rheum. 1984; 27: 873-82.

4. Beirith SC, Ikino CM, Pereira IA. Laryngeal involvement in rheumatoid arthritis. Braz J Otorhinolaryngol. 2013;79:233-8.

5. Grossman A, Martin JR, Root HS. Rheumatoid arthritis of the cricoarytenoid joint. Laryngoscope. 1961;71:530-44.

6. Rigual NR. Otolaryngologic manifestations of rheumatoid arthritis. Ear Nose Throat J. 1987; 66:18-22.

7. Hamdan AL, Sarieddine D. Laryngeal manifestations of rheumatoid arthritis. Autoimmune Dis. 2013; 103081.

8. Kiliç MA, Okur E, Yildirim I, Oğüt F, Denizoğlu I, Kizilay A et al. Reliability and validity of the Turkish version of the Voice Handicap Index. Kulak Burun Bogaz Ihtis Derg. 2008;18:139-47

9. L. G. Guerra, K. Y. Lau, and R. Marwah, "Upper airway obstruction as the sole manifestation of rheumatoid arthritis," Journal of Rheumatology, vol. 19, no. 6, pp. 974-976, 1992. [20]

10. B. Benjamin, "Laryngeal manifestations of systemic diseases," in Endolaryngeal Surgery, Martin Dunitz, London, UK, 1998.

11. Y. Hosako,M. Nakamura, N. Tayama et al., "Laryngeal involvements in systemic lupus erythematosus: a case report," Larynx, vol. 5, no. 2, pp. 171-175, 1993.

12. N. Erb, A. V. Pace, J. P. Delamere, and G. D. Kitas, "Dysphagia and stridor caused by laryngeal rheumatoid arthritis," Rheumatology, vol. 40, no. 8, pp. 952-953, 2001.

13. C. S. Darke, L. Wolman, and A. Young, "Laryngeal stridor in rheumatoid arthritis," British Medical Journal, vol. 1, pp. 1279-1282, 1958

14. HABIB MA: Intra-articular steroid injection in acute rheumatoid arthritis of the larynx. J Laryngol Otol; 91:909-910, 1977

15. P. J. S. M. Kerstens, A. M. T. Boerbooms, M. E. C. Jeurissen, J. H. Fast, K. J. M. Assmann, and L. B. A. Van De Putte, "Accelerated nodulosis during low dose methotrexate therapy for rheumatoid arthritis. An analysis of ten cases," Journal of Rheumatology, vol. 19, no. 6, pp. 867-871, 1992.

16. E. Hilgert, B. Toleti, K. Kruger, and I. Nejedlo, "Hoarseness due to bamboo nodes in patients with autoimmune diseases: a review of literature," Journal of Voice, vol. 22, no. 3, pp. 343-350, 2008

Reviewer: Assoc. prof. Dr. D. Konov

\section{Correspondence to:}

A.R.Topalova@gmail.com Aleksandrina

Topalova-Shishmanova,PhD

ENT Department, UMHAT "St. George"

Plovdiv, Medical University of Plovdiv, Bulgaria 